\title{
Stability of an Additive-Cubic-Quartic Functional Equation in Multi-Banach Spaces
}

\author{
Zhihua Wang, ${ }^{1}$ Xiaopei Li, ${ }^{2}$ and Themistocles M. Rassias ${ }^{3}$ \\ ${ }^{1}$ School of Science, Hubei University of Technology, Wuhan, Hubei 430068, China \\ ${ }^{2}$ Department of Mathematics, Zhanjiang Normal University, Zhanjiang, Guangdong 524048, China \\ ${ }^{3}$ Department of Mathematics, National Technical University of Athens, Zografou Campus, \\ 15780 Athens, Greece
}

Correspondence should be addressed to Themistocles M. Rassias, trassias@math.ntua.gr

Received 5 August 2011; Accepted 30 September 2011

Academic Editor: Narcisa C. Apreutesei

Copyright ( $) 2011$ Zhihua Wang et al. This is an open access article distributed under the Creative Commons Attribution License, which permits unrestricted use, distribution, and reproduction in any medium, provided the original work is properly cited.

\begin{abstract}
We prove the Hyers-Ulam stability of the additive-cubic-quartic functional equation in multiBanach spaces by using the fixed point alternative method. The first results on the stability in the multi-Banach spaces were presented in (Dales and Moslehian 2007).
\end{abstract}

\section{Introduction}

Stability is investigated when one is asking whether a small error of parameters in one problem causes a large deviation of its solution. Given an approximate homomorphism, is it possible to approximate it by a true homomorphism? In other words, we are looking for situations when the homomorphisms are stable, that is, if a mapping is almost a homomorphism, then there exists a true homomorphism near it with small error as much as possible. This problem was posed by Ulam in 1940 (cf. [1]) and is called the stability of functional equations. For Banach spaces, the problem was solved by Hyers [2] in the case of approximately additive mappings. Later, Hyers' result was generalized by Aoki [3] for additive mappings and by Rassias [4] for linear mappings by allowing the Cauchy difference to be unbounded. During the last decade, stability of functional equations was studied by several mathematicians for mappings in various spaces including random normed spaces and fuzzy Banach spaces (cf. $[5,6])$. For various other results on the stability of functional equations, one is referred to [7-26].

Most of the proofs of stability theorems in the Hyers-Ulam context have applied Hyers' direct method. The exact solution of the functional equation is explicitly constructed as the 
limit of a sequence, which is originating from the given approximate solution. In 2003, Radu [27] proposed the fixed point alternative method for obtaining the existence of exact solutions and error estimations and noticed that a fixed point alternative method is essential for the solution of Ulam problem for approximate homomorphisms. Subsequently, some authors $[28,29]$ applied the fixed alternative method to investigate the stability problems of several functional equations.

The notion of multi-normed space was introduced by Dales and Polyakov [30] (or see $[31,32])$. This concept is somewhat similar to operator sequence space and has some connections with operator spaces and Banach lattices. Motivations for the study of multinormed spaces and many examples were given in [30,31]. In 2007, stability of mappings on multi-normed spaces was first given in [31], and asymptotic aspect of the quadratic functional equation in multi-normed spaces was investigated in [33].

In this paper, we consider the following functional equation derived from additive, cubic, and quartic mappings:

$$
\begin{aligned}
11[f(x+2 y)+f(x-2 y)]= & 44[f(x+y)+f(x-y)]+12 f(3 y) \\
& -48 f(2 y)+60 f(y)-66 f(x) .
\end{aligned}
$$

It is easy to see that the function $f(x)=a x+b x^{3}+c x^{4}$ satisfies the functional equation (1.1). Eshaghi Gordji et al. [34] established the general solution and proved the generalized Hyers-Ulam stability for (1.1). The main purpose of this paper is to establish the Hyers-Ulam stability of (1.1) in multi-Banach spaces by using the fixed point alternative method.

\section{Preliminaries}

In this section, some useful results are pointed out. We begin with the alternative of a fixed point of Diaz and Margolis, which we will refer to as follows.

Lemma 2.1 (cf. $[27,35])$. Let $(X, d)$ be a complete generalized metric space and $J: X \rightarrow X$ be a strictly contractive mapping, that is,

$$
d(J x, J y) \leq L d(x, y) \quad \forall x, y \in X
$$

for some $L \leq 1$. Then, for each fixed element $x \in X$, either

$$
d\left(J^{n} x, J^{n+1} x\right)=+\infty \quad \forall n \geq 0,
$$

or

$$
d\left(J^{n} x, J^{n+1} x\right)<+\infty \quad \forall n \geq n_{0}
$$


for some natural number $n_{0}$. Moreover, if the second alternative holds, then

(i) the sequence $\left\{J^{n} x\right\}$ is convergent to a fixed point $y^{*}$ of $J$;

(ii) $y^{*}$ is the unique fixed point of $J$ in the set $Y:=\left\{y \in X \mid d\left(J^{n_{0}} x, y\right)<+\infty\right\}$ and $d\left(y, y^{*}\right) \leq$ $1 /(1-L) d(y, J y)$, for all $, x, y \in Y$.

Following [30, 31], we recall some basic facts concerning multi-normed spaces and some preliminary results.

Let $(\mathcal{\varepsilon},\|\cdot\|)$ be a complex normed space, and let $k \in \mathbb{N}$. We denote by $\mathcal{E}^{k}$ the linear space $\varepsilon \oplus \cdots \oplus \mathcal{\varepsilon}$ consisting of $k$-tuples $\left(x_{1}, \ldots, x_{k}\right)$, where $x_{1}, \ldots, x_{k} \in \mathcal{\varepsilon}$. The linear operations $\varepsilon^{k}$ are defined coordinatewise. The zero element of either $\varepsilon$ or $\varepsilon^{k}$ is denoted by 0 . We denote by $\mathbb{N}_{k}$ the set $\{1,2, \ldots, k\}$ and by $\mathfrak{S}_{k}$ the group of permutations on $k$ symbols.

Definition 2.2 (cf. $[30,31])$. A multi-norm on $\left\{\varepsilon^{k}: k \in \mathbb{N}\right\}$ is a sequence $\left(\|\cdot\|_{k}\right)=\left(\|\cdot\|_{k}: k \in \mathbb{N}\right)$ such that $\|\cdot\|_{k}$ is a norm on $\mathcal{\varepsilon}^{k}$ for each $k \in \mathbb{N},\|x\|_{1}=\|x\|$ for each $x \in \mathcal{E}$, and the following axioms are satisfied for each $k \in \mathbb{N}$ with $k \geq 2$ :

(N1) $\left\|\left(x_{\sigma(1)}, \ldots, x_{\sigma(k)}\right)\right\|_{k}=\left\|\left(x_{1}, \ldots, x_{k}\right)\right\|_{k}$, for $\sigma \in \mathfrak{S}_{k}, x_{1}, \ldots, x_{k} \in \mathcal{\varepsilon} ;$

(N2) $\left\|\left(\alpha_{1} x_{1}, \ldots, \alpha_{k} x_{k}\right)\right\|_{k} \leq\left(\max _{i \in \mathbb{N}_{k}}\left|\alpha_{i}\right|\right)\left\|\left(x_{1}, \ldots, x_{k}\right)\right\|_{k}$, for $\alpha_{1}, \ldots, \alpha_{k} \in \mathbb{C}, x_{1}, \ldots, x_{k} \in \mathcal{\varepsilon}$;

(N3) $\left\|\left(x_{1}, \ldots, x_{k-1}, 0\right)\right\|_{k}=\left\|\left(x_{1}, \ldots, x_{k-1}\right)\right\|_{k-1}$, for $x_{1}, \ldots, x_{k-1} \in \mathcal{\varepsilon}$;

(N4) $\left\|\left(x_{1}, \ldots, x_{k-1}, x_{k-1}\right)\right\|_{k}=\left\|\left(x_{1}, \ldots, x_{k-1}\right)\right\|_{k-1}$, for $x_{1}, \ldots, x_{k-1} \in \mathcal{\varepsilon}$.

In this case, we say that $\left(\left(\varepsilon^{k},\|\cdot\|_{k}\right): k \in \mathbb{N}\right)$ is a multi-normed space.

Suppose that $\left(\left(\varepsilon^{k},\|\cdot\|_{k}\right): k \in \mathbb{N}\right)$ is a multi-normed space, and take $k \in \mathbb{N}$. We need the following two properties of multi-norms. They can be found in [30]

(a) $\|(x, \ldots, x)\|_{k}=\|x\|$, for $x \in \mathcal{\varepsilon}$,

(b) $\max _{i \in \mathbb{N}_{k}}\left\|x_{i}\right\| \leq\left\|\left(x_{1}, \ldots, x_{k}\right)\right\|_{k} \leq \sum_{i=1}^{k}\left\|x_{i}\right\| \leq k \max _{i \in \mathbb{N}_{k}}\left\|x_{i}\right\|$, for $x_{1}, \ldots, x_{k} \in \mathcal{\varepsilon}$.

It follows from (b) that if $(\mathcal{\varepsilon},\|\cdot\|)$ is a Banach space, then $\left(\varepsilon^{k},\|\cdot\|_{k}\right)$ is a Banach space for each $k \in \mathbb{N}$; in this case, $\left(\left(\varepsilon^{k},\|\cdot\|_{k}\right): k \in \mathbb{N}\right)$ is a multi-Banach space.

Lemma 2.3 (cf. $[30,31]$ ). Suppose that $k \in \mathbb{N}$ and $\left(x_{1}, \ldots, x_{k}\right) \in \mathcal{E}^{k}$. For each $j \in\{1, \ldots, k\}$, let $\left(x_{n}^{j}\right)_{n=1,2, \ldots}$ be a sequence in $\varepsilon$ such that $\lim _{n \rightarrow \infty} x_{n}^{j}=x_{j}$. Then

$$
\lim _{n \rightarrow \infty}\left(x_{n}^{1}-y_{1}, \ldots, x_{n}^{k}-y_{k}\right)=\left(x_{1}-y_{1}, \ldots, x_{k}-y_{k}\right)
$$

holds for all $\left(y_{1}, \ldots, y_{k}\right) \in \mathcal{\varepsilon}^{k}$.

Definition 2.4 (cf. $[30,31])$. Let $\left(\left(\varepsilon^{k},\|\cdot\|_{k}\right): k \in \mathbb{N}\right)$ be a multi-normed space. A sequence $\left(x_{n}\right)$ in $\mathcal{\varepsilon}$ is a multi-null sequence if for each $\varepsilon>0$, there exists $n_{0} \in \mathbb{N}$ such that

$$
\sup _{k \in \mathbb{N}}\left\|\left(x_{n}, \ldots, x_{n+k-1}\right)\right\|_{k} \leq \varepsilon \quad\left(n \geq n_{0}\right)
$$

Let $x \in \mathcal{E}$, we say that the sequence $\left(x_{n}\right)$ is multi-convergent to $x$ in $\mathcal{\varepsilon}$ and write $\lim _{n \rightarrow \infty} x_{n}=x$ if $\left(x_{n}-x\right)$ is a multi-null sequence. 


\section{Main Results}

Throughout this section, let $\varepsilon \geq 0$, $\varepsilon$ be a linear space, and let $\left(\left(\mathcal{F}^{n},\|\cdot\|_{n}\right): n \in \mathbb{N}\right)$ be a multi-Banach space. For convenience, we use the following abbreviation for a given mapping $f: \varepsilon \rightarrow \mathcal{F}:$

$$
\begin{aligned}
D f(x, y)= & 11[f(x+2 y)+f(x-2 y)]-44[f(x+y)+f(x-y)] \\
& -12 f(3 y)+48 f(2 y)-60 f(y)+66 f(x) .
\end{aligned}
$$

Before proceeding to the proof of the main results in this section, we shall need the following two lemmas.

Lemma 3.1 (cf. [34]). If an even mapping $f: X \rightarrow Y$ satisfies (1.1), then $f$ is quartic.

Lemma 3.2 (cf. [34]). If an odd mapping $f: X \rightarrow Y$ satisfies (1.1), then $f$ is cubic-additive.

Theorem 3.3. Suppose that an even mapping $f: \varepsilon \rightarrow \mathcal{F}$ satisfies $f(0)=0$ and

$$
\sup _{k \in \mathbb{N}}\left\|\left(D f\left(x_{1}, y_{1}\right), \ldots, D f\left(x_{k}, y_{k}\right)\right)\right\|_{k} \leq \varepsilon
$$

for all $x_{1}, \ldots, x_{k}, y_{1}, \ldots, y_{k} \in \mathcal{E}$. Then there exists a unique quartic mapping $Q: \mathcal{E} \rightarrow \mathcal{F}$ satisfying (1.1) and

$$
\sup _{k \in \mathbb{N}}\left\|\left(f\left(x_{1}\right)-Q\left(x_{1}\right), \ldots, f\left(x_{k}\right)-Q\left(x_{k}\right)\right)\right\|_{k} \leq \frac{13}{330} \varepsilon
$$

for all $x_{1}, \ldots, x_{k} \in \mathcal{E}$.

Proof. Letting $x_{1}=\cdots=x_{k}=0$ in (3.2), we get

$$
\sup _{k \in \mathbb{N}}\left\|\left(-12 f\left(3 y_{1}\right)+70 f\left(2 y_{1}\right)-148 f\left(y_{1}\right), \ldots,-12 f\left(3 y_{k}\right)+70 f\left(2 y_{k}\right)-148 f\left(y_{k}\right)\right)\right\|_{k} \leq \varepsilon
$$

for all $y_{1}, \ldots, y_{k} \in \mathcal{E}$. Replacing $x_{1}, \ldots, x_{k}$ with $y_{1}, \ldots, y_{k}$ in (3.2), we obtain

$$
\sup _{k \in \mathbb{N}}\left\|\left(-f\left(3 y_{1}\right)+4 f\left(2 y_{1}\right)+17 f\left(y_{1}\right), \ldots,-f\left(3 y_{k}\right)+4 f\left(2 y_{k}\right)+17 f\left(y_{k}\right)\right)\right\|_{k} \leq \varepsilon
$$

for all $y_{1}, \ldots, y_{k} \in \mathcal{\varepsilon}$.

It follows from (3.4) and (3.5) that

$$
\sup _{k \in \mathbb{N}}\left\|\left(f\left(2 x_{1}\right)-16 f\left(x_{1}\right), \ldots, f\left(2 x_{k}\right)-16 f\left(x_{k}\right)\right)\right\|_{k} \leq \frac{13}{22} \varepsilon
$$

for all $x_{1}, \ldots, x_{k} \in \mathcal{\varepsilon}$. 
by

Let $E:=\{g g: \varepsilon \rightarrow \mathcal{F}, g(0)=0\}$, and introduce the generalized metric $d$ defined on $E$

$$
d(g, h)=\inf \left\{c \in(0, \infty) \mid \sup _{k \in \mathbb{N}}\left\|\left(g\left(x_{1}\right)-h\left(x_{1}\right), \ldots, g\left(x_{k}\right)-h\left(x_{k}\right)\right)\right\|_{k} \leq c, \forall x_{1}, \ldots, x_{k} \in \varepsilon\right\}
$$

Then, it is easy to show that $d$ is a complete generalized metric on $E$ (see the proof in [36] or [5]). We now define a function $J_{1}: E \rightarrow E$ by

$$
J_{1} g(x)=\frac{1}{16} g(2 x), \quad \forall x \in \varepsilon
$$

We assert that $J_{1}$ is a strictly contractive mapping. Given $g, h \in E$, let $c \in(0, \infty)$ be an arbitrary constant with $d(g, h) \leq c$. From the definition of $d$, it follows that

$$
\sup _{k \in \mathbb{N}}\left\|\left(g\left(x_{1}\right)-h\left(x_{1}\right), \ldots, g\left(x_{k}\right)-h\left(x_{k}\right)\right)\right\|_{k} \leq c
$$

for all $x_{1}, \ldots, x_{k} \in \mathcal{E}$. Therefore,

$$
\begin{aligned}
\sup _{k \in \mathbb{N}} \| & \left(J_{1} g\left(x_{1}\right)-J_{1} h\left(x_{1}\right), \ldots, J_{1} g\left(x_{k}\right)-J_{1} h\left(x_{k}\right)\right) \|_{k} \\
= & \sup _{k \in \mathbb{N}}\left\|\left(\frac{1}{16} g\left(2 x_{1}\right)-\frac{1}{16} h\left(2 x_{1}\right), \ldots, \frac{1}{16} g\left(2 x_{k}\right)-\frac{1}{16} h\left(2 x_{k}\right)\right)\right\|_{k} \leq \frac{1}{16} c
\end{aligned}
$$

for all $x_{1}, \ldots, x_{k} \in \mathcal{E}$. Hence, it holds that $d\left(J_{1} g, J_{1} h\right) \leq(1 / 16) c$, that is, $d\left(J_{1} g, J_{1} h\right) \leq$ $(1 / 16) d(g, h)$ for all $g, h \in E$.

By using (3.6), we have $d\left(J_{1} f, f\right) \leq(13 / 352) \varepsilon$. According to Lemma 2.1, we deduce the existence of a fixed point of $J_{1}$, that is, the existence of a mapping $Q: X \rightarrow Y$ such that $Q(2 x)=16 Q(x)$ for all $x \in \mathcal{\varepsilon}$. Moreover, we have $d\left(J_{1}^{n} f, Q\right) \rightarrow 0$, which implies that

$$
Q(x)=\lim _{n \rightarrow \infty}\left(J_{1}^{n} f\right)(x)=\lim _{n \rightarrow \infty} \frac{f\left(2^{n} x\right)}{16^{n}}, \quad \forall x \in \mathcal{\varepsilon}
$$

Also, $d(f, Q) \leq 1 /(1-L) d\left(J_{1} f, f\right)$ implies the inequality

$$
d(f, Q) \leq \frac{1}{1-(1 / 16)} d\left(J_{1} f, f\right) \leq \frac{13}{330} \varepsilon
$$


Set $x_{1}=\cdots=x_{k}=2^{n} x, y_{1}=\cdots=y_{k}=2^{n} y$ in (3.2), and divide both sides by $16^{n}$. Then, using property $(a)$, we obtain

$$
\|D Q(x, y)\|=\lim _{n \rightarrow \infty} \frac{1}{16^{n}}\left\|D f\left(2^{n} x, 2^{n} y\right)\right\| \leq \lim _{n \rightarrow \infty} \frac{\varepsilon}{16^{n}}=0
$$

for all $x, y \in \mathcal{E}$. Hence, by Lemma 3.1, $Q$ is quartic.

The uniqueness of $Q$ follows from the fact that $Q$ is the unique fixed point of $J_{1}$ with the property that there exists $l \in(0, \infty)$ such that

$$
\sup _{k \in \mathbb{N}}\left\|\left(f\left(x_{1}\right)-Q\left(x_{1}\right), \ldots, f\left(x_{k}\right)-Q\left(x_{k}\right)\right)\right\|_{k} \leq l
$$

for all $x_{1}, \ldots, x_{k} \in \mathcal{E}$. This completes the proof of the theorem.

Theorem 3.4. Suppose that an odd mapping $f: \varepsilon \rightarrow \mathcal{F}$ satisfies

$$
\sup _{k \in \mathbb{N}}\left\|\left(D f\left(x_{1}, y_{1}\right), \ldots, D f\left(x_{k}, y_{k}\right)\right)\right\|_{k} \leq \varepsilon
$$

for all $x_{1}, \ldots, x_{k}, y_{1}, \ldots, y_{k} \in \mathcal{E}$. Then there exists a unique additive mapping $A: \mathcal{E} \rightarrow \mathcal{F}$ and $a$ unique cubic mapping $C: \varepsilon \rightarrow F$ satisfying (1.1) and

$$
\begin{aligned}
& \sup _{k \in \mathbb{N}}\left\|\left(f\left(2 x_{1}\right)-8 f\left(x_{1}\right)-A\left(x_{1}\right), \ldots, f\left(2 x_{k}\right)-8 f\left(x_{k}\right)-A\left(x_{k}\right)\right)\right\|_{k} \leq \frac{17}{33} \varepsilon, \\
& \sup _{k \in \mathbb{N}}\left\|\left(f\left(2 x_{1}\right)-2 f\left(x_{1}\right)-C\left(x_{1}\right), \ldots, f\left(2 x_{k}\right)-2 f\left(x_{k}\right)-C\left(x_{k}\right)\right)\right\|_{k} \leq \frac{17}{231} \varepsilon
\end{aligned}
$$

for all $x_{1}, \ldots, x_{k} \in \mathcal{\varepsilon}$.

Proof. Put $x_{1}=\cdots=x_{k}=0$ in (3.15). Then, by the oddness of $f$, we have

$$
\sup _{k \in \mathbb{N}}\left\|\left(12 f\left(3 y_{1}\right)-48 f\left(2 y_{1}\right)+60 f\left(y_{1}\right), \ldots 12 f\left(3 y_{k}\right)-48 f\left(2 y_{k}\right)+60 f\left(y_{k}\right)\right)\right\|_{k} \leq \varepsilon
$$

for all $y_{1}, \ldots, y_{k} \in \mathcal{E}$. Replacing $x_{1}, \ldots, x_{k}$ with $2 y_{1}, \ldots, 2 y_{k}$ in (3.15), we obtain

$$
\begin{aligned}
\sup _{k \in \mathbb{N}} \|\left(11 f\left(4 y_{1}\right)-56 f\left(3 y_{1}\right)+114 f\left(2 y_{1}\right)-104 f\left(y_{1}\right), \ldots, 11 f\left(4 y_{k}\right)-56 f\left(3 y_{k}\right)\right. & \\
+ & \left.114 f\left(2 y_{k}\right)-104 f\left(y_{k}\right)\right) \|_{k} \leq \varepsilon
\end{aligned}
$$

for all $y_{1}, \ldots, y_{k} \in \mathcal{E}$. By (3.17) and (3.18), we have

$$
\sup _{k \in \mathbb{N}}\left\|\left(f\left(4 x_{1}\right)-10 f\left(2 x_{1}\right)+16 f\left(x_{1}\right), \ldots, f\left(4 x_{k}\right)-10 f\left(2 x_{k}\right)+16 f\left(x_{k}\right)\right)\right\|_{k} \leq \frac{17}{33} \varepsilon
$$


for all $x_{1}, \ldots, x_{k} \in \mathcal{E}$. Putting $\alpha(x):=f(2 x)-8 f(x)$ for all $x \in \mathcal{E}$, we get

$$
\sup _{k \in \mathbb{N}}\left\|\left(\alpha\left(2 x_{1}\right)-2 \alpha\left(x_{1}\right), \ldots, \alpha\left(2 x_{k}\right)-2 \alpha\left(x_{k}\right)\right)\right\|_{k} \leq \frac{17}{33} \varepsilon
$$

for all $x_{1}, \ldots, x_{k} \in \mathcal{\varepsilon}$.

Let the same definitions for $E$ and $d$ as in the proof of Theorem 3.3 such that $(E, d)$ becomes a complete generalized metric space. We now define a function $J_{2}: E \rightarrow E$ by

$$
J_{2} g(x)=\frac{1}{2} g(2 x), \quad \forall x \in \mathcal{\varepsilon} .
$$

Applying a similar technique as in the proof of Theorem 3.3, we obtain $d\left(J_{2} g, J_{2} h\right) \leq(1 / 2) c$, that is, $d\left(J_{2} g, J_{2} h\right) \leq(1 / 2) d(g, h)$ for all $g, h \in E$.

By (3.20), we have $d\left(J_{2} \alpha, \alpha\right) \leq(17 / 66) \varepsilon$. According to Lemma 2.1, we deduce the existence of a fixed point of $J_{2}$, that is, the existence of a mapping $A: X \rightarrow Y$ such that $A(2 x)=2 A(x)$ for all $x \in \mathcal{E}$. Moreover, we have $d\left(J_{2}^{n} \alpha, A\right) \rightarrow 0$, which implies that

$$
A(x)=\lim _{n \rightarrow \infty}\left(J_{2}^{n} \alpha\right)(x)=\lim _{n \rightarrow \infty} \frac{\alpha\left(2^{n} x\right)}{2^{n}}, \quad \forall x \in \mathcal{\varepsilon}
$$

Also, $d(\alpha, A) \leq 1 /(1-L) d\left(J_{2} \alpha, \alpha\right)$ implies the inequality

$$
d(\alpha, A) \leq \frac{1}{1-(1 / 2)} d\left(J_{2} \alpha, \alpha\right) \leq \frac{17}{33} \varepsilon
$$

Hence, it follows that

$$
\begin{aligned}
\|D A(x, y)\| & =\lim _{n \rightarrow \infty} \frac{1}{2^{n}}\left\|D \alpha\left(2^{n} x, 2^{n} y\right)\right\| \\
& =\lim _{n \rightarrow \infty} \frac{1}{2^{n}}\left\|D f\left(2^{n+1} x, 2^{n+1} y\right)-8 D f\left(2^{n} x, 2^{n} y\right)\right\| \leq \lim _{n \rightarrow \infty} \frac{\varepsilon}{2^{n}}=0
\end{aligned}
$$

for all $x, y \in \mathcal{E}$. This means that $A$ satisfies (1.1). Then, by Lemma 3.2, $x \mapsto A(2 x)-8 A(x)$ is additive. Thus, by $A(2 x)=2 A(x)$, we conclude that $A$ is additive.

Putting $\beta(x):=f(2 x)-2 f(x)$ for all $x \in \mathcal{E}$, we get

$$
\sup _{k \in \mathbb{N}}\left\|\left(\beta\left(2 x_{1}\right)-8 \beta\left(x_{1}\right), \ldots, \alpha\left(2 x_{k}\right)-8 \beta\left(x_{k}\right)\right)\right\|_{k} \leq \frac{17}{33} \varepsilon
$$

for all $x_{1}, \ldots, x_{k} \in \mathcal{\varepsilon}$. We now define a function $J_{3}: E \rightarrow E$ by

$$
J_{3} g(x)=\frac{1}{8} g(2 x), \quad \forall x \in \mathcal{\varepsilon} .
$$


Applying a similar technique as in the proof of Theorem 3.3, we obtain $d\left(J_{3} g, J_{3} h\right) \leq(1 / 8) c$, that is, $d\left(J_{3} g, J_{3} h\right) \leq(1 / 8) d(g, h)$ for all $g, h \in E$.

By (3.25), we have $d\left(J_{3} \beta, \beta\right) \leq(17 / 264) \varepsilon$. According to Lemma 2.1 , we deduce the existence of a fixed point of $J_{3}$, that is, the existence of a mapping $C: X \rightarrow Y$ such that $C(2 x)=8 C(x)$ for all $x \in \mathcal{E}$. Moreover, we have $d\left(J_{3}^{n} \beta, C\right) \rightarrow 0$, which implies that

$$
C(x)=\lim _{n \rightarrow \infty}\left(J_{3}^{n} \beta\right)(x)=\lim _{n \rightarrow \infty} \frac{\beta\left(2^{n} x\right)}{8^{n}}, \quad \forall x \in \mathcal{\varepsilon}
$$

Also, $d(\beta, C) \leq 1 /(1-L) d\left(J_{3} \beta, \beta\right)$ implies the inequality

$$
d(\beta, C) \leq \frac{1}{1-(1 / 8)} d\left(J_{3} \beta, \beta\right) \leq \frac{17}{231} \varepsilon
$$

Then we have

$$
\begin{aligned}
\|D C(x, y)\| & =\lim _{n \rightarrow \infty} \frac{1}{8^{n}}\left\|D \beta\left(2^{n} x, 2^{n} y\right)\right\| \\
& =\lim _{n \rightarrow \infty} \frac{1}{8^{n}}\left\|D f\left(2^{n+1} x, 2^{n+1} y\right)-2 D f\left(2^{n} x, 2^{n} y\right)\right\| \leq \lim _{n \rightarrow \infty} \frac{\varepsilon}{8^{n}}=0
\end{aligned}
$$

for all $x, y \in \mathcal{E}$. Hence, the mapping $C$ satisfies (1.1). Therefore, by Lemma 3.2, $x \mapsto C(2 x)-$ $2 C(x)$ is cubic. Thus, $C(2 x)=8 C(x)$ implies that the mapping $C$ is cubic.

The uniqueness of $A$ and $C$ can be proved in the same reasoning as in the proof of Theorem 3.3. This completes the proof of the theorem.

Theorem 3.5. Suppose that an odd mapping $f: \varepsilon \rightarrow \mathcal{F}$ satisfies

$$
\sup _{k \in \mathbb{N}}\left\|\left(D f\left(x_{1}, y_{1}\right), \ldots, D f\left(x_{k}, y_{k}\right)\right)\right\|_{k} \leq \varepsilon
$$

for all $x_{1}, \ldots, x_{k}, y_{1}, \ldots, y_{k} \in \mathcal{E}$. Then there exists a unique additive mapping $A: \mathcal{E} \rightarrow \mathcal{F}$ and $a$ unique cubic mapping $C: \mathcal{E} \rightarrow \mathcal{F}$ such that

$$
\sup _{k \in \mathbb{N}}\left\|\left(f\left(x_{1}\right)-A\left(x_{1}\right)-C\left(x_{1}\right), \ldots, f\left(x_{k}\right)-A\left(x_{k}\right)-C\left(x_{k}\right)\right)\right\|_{k} \leq \frac{68}{693} \varepsilon
$$

for all $x_{1}, \ldots, x_{k} \in \mathcal{\varepsilon}$.

Proof. By Theorem 3.4, there exist an additive mapping $A_{0}: \mathcal{E} \rightarrow \mathcal{F}$ and a cubic mapping $C_{0}: \varepsilon \rightarrow F$ such that

$$
\begin{aligned}
& \sup _{k \in \mathbb{N}}\left\|\left(f\left(2 x_{1}\right)-8 f\left(x_{1}\right)-A_{0}\left(x_{1}\right), \ldots, f\left(2 x_{k}\right)-8 f\left(x_{k}\right)-A_{0}\left(x_{k}\right)\right)\right\|_{k} \leq \frac{17}{33} \varepsilon, \\
& \sup _{k \in \mathbb{N}}\left\|\left(f\left(2 x_{1}\right)-2 f\left(x_{1}\right)-C_{0}\left(x_{1}\right), \ldots, f\left(2 x_{k}\right)-2 f\left(x_{k}\right)-C_{0}\left(x_{k}\right)\right)\right\|_{k} \leq \frac{17}{231} \varepsilon
\end{aligned}
$$


for all $x_{1}, \ldots, x_{k} \in \mathcal{\varepsilon}$. Combining the above two equations in (3.32) yields that

$$
\sup _{k \in \mathbb{N}}\left\|\left(6 f\left(x_{1}\right)+A_{0}\left(x_{1}\right)-C_{0}\left(x_{1}\right), \ldots, f\left(6 x_{k}\right)+A_{0}\left(x_{k}\right)-C_{0}\left(x_{k}\right)\right)\right\|_{k} \leq \frac{136}{231} \varepsilon
$$

for all $x_{1}, \ldots, x_{k} \in \mathcal{E}$. So we obtain (3.31) by letting $A=-A_{0} / 6$ and $C=C_{0} / 6$.

To prove the uniqueness of $A$ and $C$, let $A_{1}, C_{1}: \varepsilon \rightarrow \mp$ be other additive and cubic mappings satisfying (3.31). Let $A^{\prime}=A-A_{1}$ and $C^{\prime}=C-C_{1}$. Then, using property $(a)$, we obtain

$$
\left\|A^{\prime}(x)+C^{\prime}(x)\right\| \leq\|f(x)-A(x)-C(x)\|+\left\|f(x)-A_{1}(x)-C_{1}(x)\right\| \leq \frac{136}{693} \varepsilon
$$

for all $x \in \mathcal{E}$, then (3.34) implies that

$$
\lim _{n \rightarrow \infty} \frac{1}{8^{n}}\left\|A^{\prime}\left(2^{n} x\right)+C^{\prime}\left(2^{n} x\right)\right\|=0
$$

for all $x \in \mathcal{E}$. Therefore, $C^{\prime}(x)=0$ for all $x \in \mathcal{E}$. By (3.34), we have $A^{\prime}(x)=0$ for all $x \in \mathcal{\varepsilon}$. This completes the proof of the theorem.

Theorem 3.6. Suppose that a mapping $f: \mathcal{E} \rightarrow \mathcal{F}$ satisfies $f(0)=0$ and

$$
\sup _{k \in \mathbb{N}}\left\|\left(D f\left(x_{1}, y_{1}\right), \ldots, D f\left(x_{k}, y_{k}\right)\right)\right\|_{k} \leq \varepsilon
$$

for all $x_{1}, \ldots, x_{k}, y_{1}, \ldots, y_{k} \in \mathcal{E}$. Then there exists a unique additive mapping $A: \mathcal{E} \rightarrow \mathcal{F}$, a unique cubic mapping $C: \mathcal{E} \rightarrow \mathcal{F}$, and a unique quartic mapping $Q: \mathcal{E} \rightarrow \mathcal{F}$ such that

$$
\sup _{k \in \mathbb{N}} \|\left(f\left(x_{1}\right)-A\left(x_{1}\right)-C\left(x_{1}-Q\left(x_{1}\right), \ldots, f\left(x_{k}\right)-A\left(x_{k}\right)-C\left(x_{k}\right)-Q\left(x_{k}\right)\right) \|_{k} \leq \frac{953}{6930} \varepsilon\right.
$$

for all $x_{1}, \ldots, x_{k} \in \mathcal{E}$.

Proof. Let $f_{o}(x)=(1 / 2)(f(x)-f(-x))$ for all $x \in \mathcal{E}$, then $f_{o}(0)=0, f_{o}(-x)=-f_{o}(x)$ and

$$
\sup _{k \in \mathbb{N}}\left\|\left(D f_{o}\left(x_{1}, y_{1}\right), \ldots, D f_{o}\left(x_{k}, y_{k}\right)\right)\right\|_{k} \leq \varepsilon
$$

for all $x_{1}, \ldots, x_{k}, y_{1}, \ldots, y_{k} \in \mathcal{E}$. From Theorem 3.5, it follows that there exists a unique additive mapping $A: \mathcal{\varepsilon} \rightarrow \mathcal{F}$ and a unique cubic mapping $C: \mathcal{E} \rightarrow \mathcal{F}$ satisfying (3.31).

Let $f_{e}(x)=(1 / 2)(f(x)+f(-x))$ for all $x \in \mathcal{E}$, then $f_{e}(0)=0, f_{e}(-x)=f_{e}(x)$ and

$$
\sup _{k \in \mathbb{N}}\left\|\left(D f_{e}\left(x_{1}, y_{1}\right), \ldots, D f_{e}\left(x_{k}, y_{k}\right)\right)\right\|_{k} \leq \varepsilon
$$


for all $x_{1}, \ldots, x_{k}, y_{1}, \ldots, y_{k} \in \mathcal{E}$. By Theorem 3.3, there exists a unique quartic mapping $Q: \mathcal{E} \rightarrow \mathcal{F}$ satisfying (3.3). Now it is obvious that (3.37) holds for all $x_{1}, \ldots, x_{k} \in \mathcal{E}$. This completes the proof of the theorem.

\section{Acknowledgments}

The authors are very grateful to the referees for their helpful comments and suggestions. This research is supported by Guangdong Provincial Natural Science Foundation (07301595), China.

\section{References}

[1] S. M. Ulam, Problems in Modern Mathematics, John Wiley \& Sons, New York, NY, USA, 1964.

[2] D. H. Hyers, "On the stability of the linear functional equation," Proceedings of the National Academy of Sciences of the United States of America, vol. 27, pp. 222-224, 1941.

[3] T. Aoki, "On the stability of the linear transformation in Banach spaces," Journal of the Mathematical Society of Japan, vol. 2, pp. 64-66, 1950.

[4] Th. M. Rassias, "On the stability of the linear mapping in Banach spaces," Proceedings of the American Mathematical Society, vol. 72, no. 2, pp. 297-300, 1978.

[5] D. Miheț and V. Radu, "On the stability of the additive Cauchy functional equation in random normed spaces," Journal of Mathematical Analysis and Applications, vol. 343, no. 1, pp. 567-572, 2008.

[6] A. K. Mirmostafaee and M. S. Moslehian, "Fuzzy versions of Hyers-Ulam-Rassias theorem," Fuzzy Sets and Systems, vol. 159, no. 6, pp. 720-729, 2008.

[7] P. W. Cholewa, "Remarks on the stability of functional equations," Aequationes Mathematicae, vol. 27, no. 1-2, pp. 76-86, 1984.

[8] St. Czerwik, "On the stability of the quadratic mapping in normed spaces," Abhandlungen aus dem Mathematischen Seminar der Universität Hamburg, vol. 62, pp. 59-64, 1992.

[9] S. Czerwik, "The stability of the quadratic functional equation," in Stability of Mappings of Hyers-Ulam Type, Th. M. Rassias and J. Tabor, Eds., Hadronic Press Collect. Orig. Artic., pp. 81-91, Hadronic Press, Palm Harbor, Fla, USA, 1994.

[10] S. Czerwik, Stability of Functional Equations of Ulam-Hyers-Rassias Type, Hadronic Press, Palm Harbor, Fla, USA, 2003.

[11] V. Faĭziev and Th. M. Rassias, “The space of $(\psi, \gamma)$-pseudocharacters on semigroups," Nonlinear Functional Analysis and Applications, vol. 5, no. 1, pp. 107-126, 2000.

[12] V. A. Fă̌ziev, Th. M. Rassias, and P. K. Sahoo, "The space of $(\psi, \gamma)$-additive mappings on semigroups," Transactions of the American Mathematical Society, vol. 354, no. 11, pp. 4455-4472, 2002.

[13] P. Găvruță, S.-M. Jung, and Y. Li, "Hyers-Ulam stability of mean value points," Annals of Functional Analysis, vol. 1, no. 2, pp. 68-74, 2010.

[14] A. Gilányi, K. Nagatou, and P. Volkmann, "Stability of a functional equation coming from the characterization of the absolute value of additive functions," Annals of Functional Analysis, vol. 1, no. 2, pp. 1-6, 2010.

[15] D. H. Hyers and Th. M. Rassias, "Approximate homomorphisms," Aequationes Mathematicae, vol. 44, no. 2-3, pp. 125-153, 1992.

[16] G. Isac and Th. M. Rassias, "On the Hyers-Ulam stability of $\psi$-additive mappings," Journal of Approximation Theory, vol. 72, no. 2, pp. 131-137, 1993.

[17] G. Isac and Th. M. Rassias, "Stability of $\psi$-additive mappings: applications to nonlinear analysis," International Journal of Mathematics and Mathematical Sciences, vol. 19, no. 2, pp. 219-228, 1996.

[18] S.-M. Jung, Hyers-Ulam-Rassias Stability of Functional Equations in Mathematical Analysis, Hadronic Press, Palm Harbor, Fla, USA, 2001.

[19] S.-M. Jung, Hyers-Ulam-Rassias Stability of Functional Equations in Nonlinear Analysis, vol. 48 of Springer Optimization and Its Applications, Springer, New York, NY, USA, 2011.

[20] K.-W. Jun and H.-M. Kim, "The generalized Hyers-Ulam-Rassias stability of a cubic functional equation," Journal of Mathematical Analysis and Applications, vol. 274, no. 2, pp. 267-278, 2002.

[21] S. H. Lee, S. M. Im, and I. S. Hwang, "Quartic functional equations," Journal of Mathematical Analysis and Applications, vol. 307, no. 2, pp. 387-394, 2005. 
[22] Th. M. Rassias, "On a modified Hyers-Ulam sequence," Journal of Mathematical Analysis and Applications, vol. 158, no. 1, pp. 106-113, 1991.

[23] Th. M. Rassias, "On the stability of functional equations in Banach spaces," Journal of Mathematical Analysis and Applications, vol. 251, no. 1, pp. 264-284, 2000.

[24] Th. M. Rassias, "On the stability of functional equations originated by a problem of Ulam," Mathematica, vol. 44(67), no. 1, pp. 39-75, 2002.

[25] Th. M. Rassias, "On the stability of minimum points," Mathematica, vol. 45(68), no. 1, pp. 93-104, 2003.

[26] Th. M. Rassias, Ed., Functional Equations, Inequalities and Applications, Kluwer Academic Publishers, Dordrecht, The Netherlands, 2003.

[27] V. Radu, "The fixed point alternative and the stability of functional equations," Fixed Point Theory, vol. 4, no. 1, pp. 91-96, 2003.

[28] L. P. Castro and A. Ramos, "Hyers-Ulam-Rassias stability for a class of nonlinear Volterra integral equations," Banach Journal of Mathematical Analysis, vol. 3, no. 1, pp. 36-43, 2009.

[29] C. Park and Th. M. Rassias, "Fixed points and generalized Hyers-Ulam stability of quadratic functional equations," Journal of Mathematical Inequalities, vol. 1, no. 4, pp. 515-528, 2007.

[30] H. G. Dales and M. E. Polyakov, "Multi-normed spaces and multi-Banach algebras," preprint.

[31] H. G. Dales and M. S. Moslehian, "Stability of mappings on multi-normed spaces," Glasgow Mathematical Journal, vol. 49, no. 2, pp. 321-332, 2007.

[32] M. S. Moslehian, "Superstability of higher derivations in multi-Banach algebras," Tamsui Oxford Journal of Mathematical Sciences, vol. 24, no. 4, pp. 417-427, 2008.

[33] M. S. Moslehian, K. Nikodem, and D. Popa, "Asymptotic aspect of the quadratic functional equation in multi-normed spaces," Journal of Mathematical Analysis and Applications, vol. 355, no. 2, pp. 717-724, 2009.

[34] M. Eshaghi-Gordji, S. Kaboli-Gharetapeh, C. Park, and S. Zolfaghari, "Stability of an additive-cubicquartic functional equation," Advances in Difference Equations, vol. 2009, Article ID 395693, 20 pages, 2009.

[35] J. B. Diaz and B. Margolis, "A fixed point theorem of the alternative, for contractions on a generalized complete metric space," Bulletin of the American Mathematical Society, vol. 74, pp. 305-309, 1968.

[36] O. Hadžić, E. Pap, and V. Radu, "Generalized contraction mapping principles in probabilistic metric spaces," Acta Mathematica Hungarica, vol. 101, no. 1-2, pp. 131-148, 2003. 


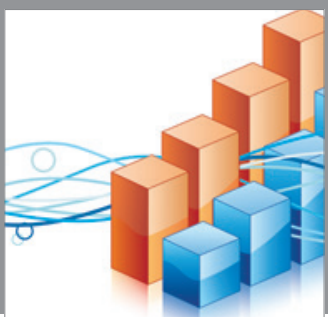

Advances in

Operations Research

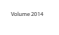

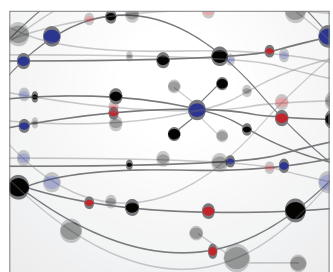

\section{The Scientific} World Journal
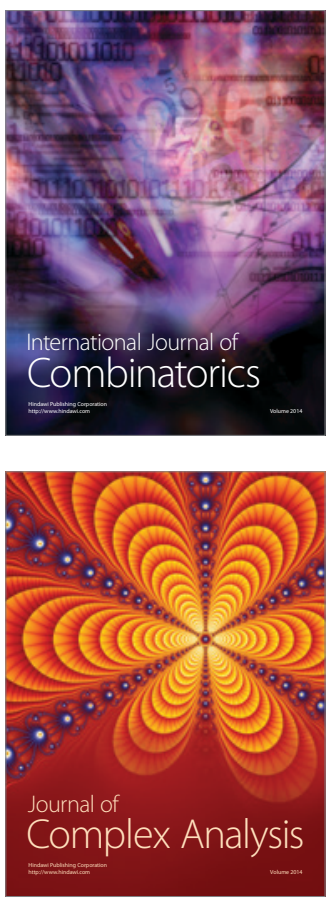

International Journal of

Mathematics and

Mathematical

Sciences
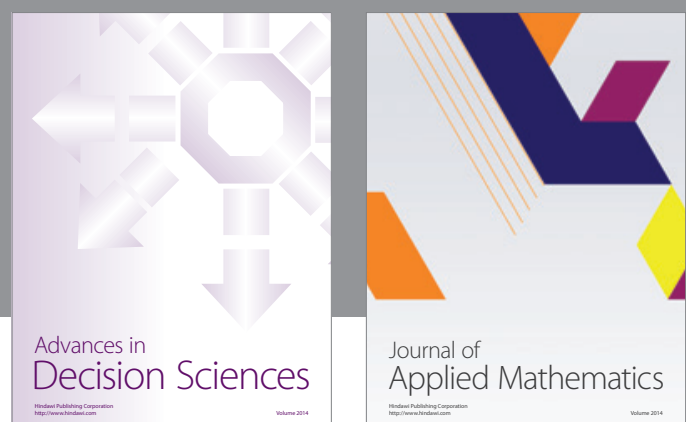

Journal of

Applied Mathematics
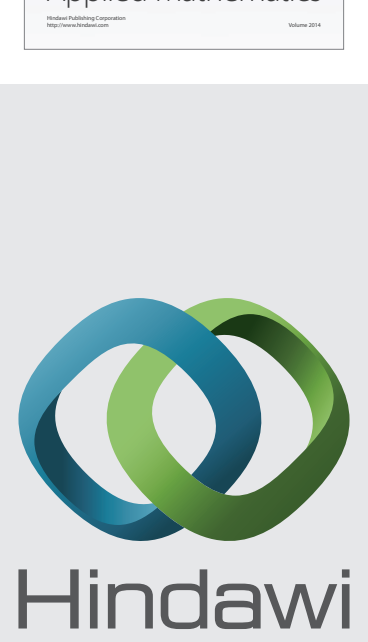

Submit your manuscripts at http://www.hindawi.com
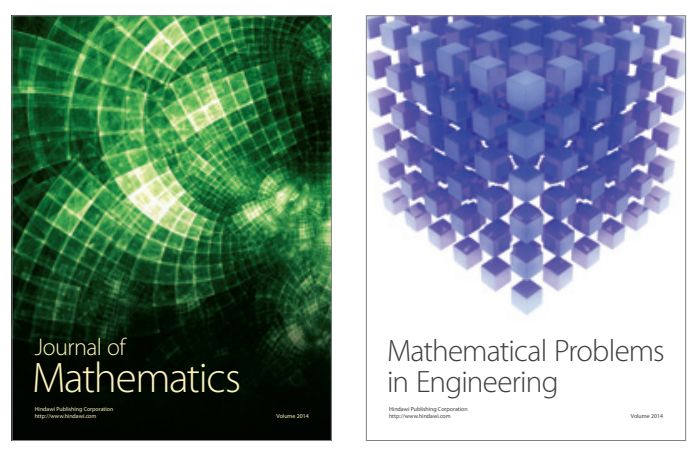

Mathematical Problems in Engineering
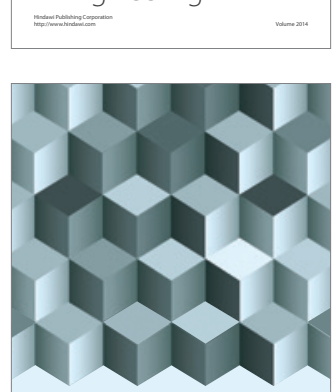

Journal of

Function Spaces
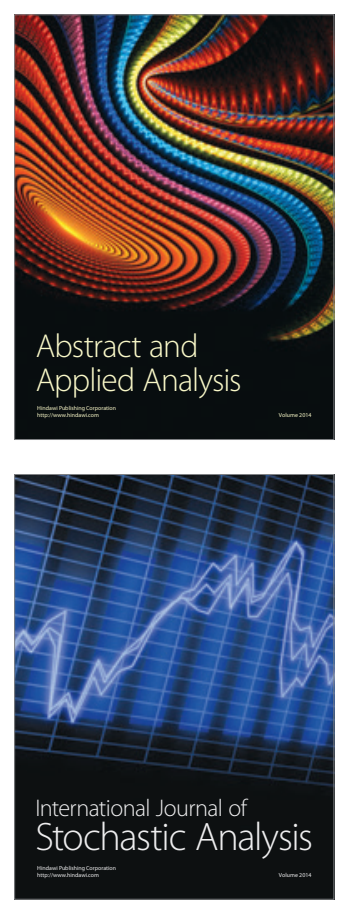

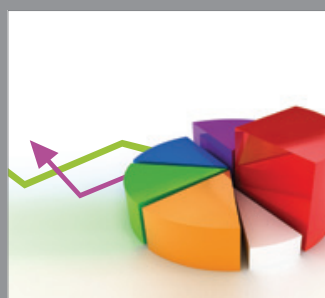

ournal of

Probability and Statistics

Promensencen
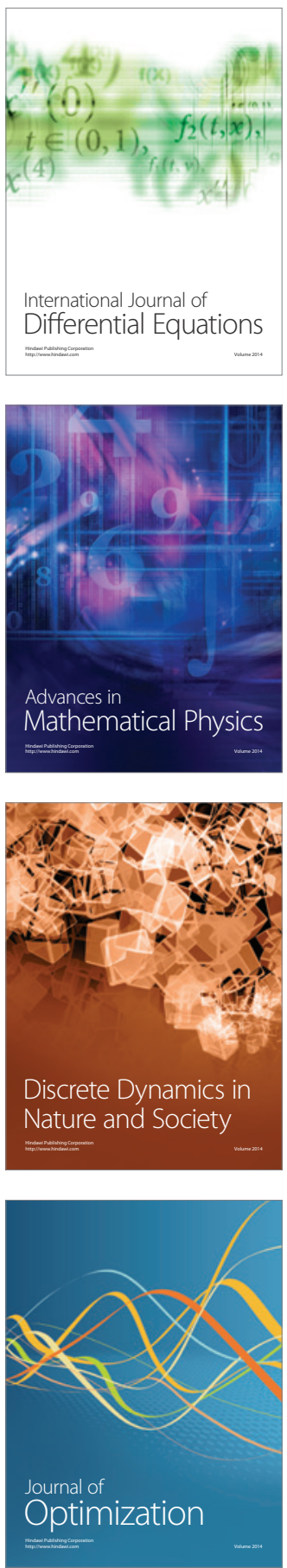